\title{
P1F.2 SENSITIVITY OF SIMULATED TROPICAL CYCLONE STRUCTURE AND INTENSITY TO CHANGES IN HORIZONTAL RESOLUTION
}

\author{
Megan S. Gentry ${ }^{+}$and Gary M. Lackmann
}

North Carolina State University

\section{INTRODUCTION}

Both human forecasters and numerical models have struggled to achieve consistent improvement in tropical cyclone (TC) intensity prediction; in contrast, TC track error has been steadily decreasing for several years (Rogers et al. 2006). Previous studies have provided multiple reasons for the lack of improvement in the forecasting of intensity. Rogers et al. (2006) cite inadequate computational resources to run operational models at sufficiently high spatial resolution, along with incomplete representation of important physical processes. Therefore, as higher resolution model runs become possible, it is vital to understand how the simulation of physical processes, especially those important to TC intensification, are impacted by changes in horizontal grid spacing. The objective of this study is to analyze simulations of Hurricane Ivan (2004) to determine sensitivity of TC intensity and structure to grid spacing between 10 to $1 \mathrm{~km}$. Ultimately, we wish to identify the requisite resolution for adequate TC intensity prediction.

\section{BACKGROUND}

In previous studies of sensitivity of convective systems to horizontal resolution, significant changes in precipitation and vertical motion are observed (Weisman et al. 1997; Bryan et al. 2003). Weisman et al. simulated squall lines to test the sensitivity of horizontal grid resolution on explicit convection, using grid spacings between 1 and $12 \mathrm{~km}$. This study concluded that $4 \mathrm{~km}$ grid spacing could reproduce essential features of the structure and evolution of the squall line seen in a $1 \mathrm{~km}$ run. Bryan et al. (2003) focus on grid spacings below $1-\mathrm{km}$ and recommend grid spacings on the order of $100 \mathrm{~m}$ to be used as a benchmark in resolution sensitivity studies in order to better represent intracloud motions.

\footnotetext{
${ }^{+}$Corresponding author address: Megan Gentry, North Carolina State University, Department of Marine, Earth and Atmospheric Sciences, Raleigh, NC 27695-8208. E-mail: msgentry@ncsu.edu
}

This study found no systematic trend in mean vertical velocity; and, instead, found that differences between the model runs evolved from the ability of the higher-resolution runs to become turbulent. Although the characteristics of a squall line updraft are significantly different than the updraft of a TC eyewall, some conclusions of these studies are applicable to TC updrafts. It might be expected that the magnitude of vertical motions would increase as smaller grid spacing is used, as updrafts are more adequately resolved. But from Bryan's conclusion, it also follows that the use of increasingly higher resolution could eventually weaken the magnitude of vertical motions, because detrimental processes, such as entrainment of lower momentum air, might begin to be somewhat resolved (Bryan, personal communication).

While these studies concentrate on resolution sensitivity in a squall line, the current study is concerned with how changes in grid spacing will affect processes important to TC intensification. In the axisymmetric conception of TC intensification, the ability of the system to deepen depends on the ability of the radial circulation to supply turbulent fluxes of heat and moisture (Ooyama 1982; Emanuel 1986; Rotunno and Emanuel 1987). The azimuthal circulation maintains the inertial stability of the system, keeping the warm-core vortex vertically aligned over the surface low pressure and maintaining the surface confluence of fluxes (Ooyama 1982). However, asymmetric, intra-eyewall physical processes also play a significant role in determining TC structure and intensity (e.g., Yang et al. 2007).

Vortex Rossby waves (VRWs) have been theorized to play an important role in TC intensity, mixing low-momentum, high potential temperature $(\theta)$ air from the eyewall into the eye (e.g., Schubert et al. 1999). This high- $\theta$ air could act as fuel for buoyant updrafts cores, which would augment the mass transport of the symmetric moist-neutral ascent, bringing about a 
state of superintensity, or intensity surpassing that predicted from symmetrical processes (Persing and Montgomery 2003). Cram et al (2007) used a 2-km simulation of Hurricane Bonnie (1998) and Lagrangian trajectories to show parcels mixing from the eye into the eyewall, where the eye contained a reservoir of high equivalent $\theta\left(\theta_{\mathrm{e}}\right)$ air because of turbulent fluxes from the ocean's surface.

\section{METHODS}

The Weather Research and Forecasting (WRF) model (Version 2.2) is used for four different simulations, all of which have the same physics options implemented, with no convective parameterization $(\mathrm{CP})$ used on the inner, higherresolution nests. The WSM 6-class graupel microphysics parameterization and the MellorYamada-Janjic (Janjic 1994, 2002) planetary boundary layer scheme are used on all domains for all runs. All domains are initialized on 11 September 2004 at 0000 UTC using Global Forecast System (GFS) initial conditions and 0.5 degree Real-Time Global sea-surface temperature analysis (Thiebaux et al. 2003). The Kain-Fritsch (KF; Kain and Fritsch 1993) CP scheme is employed on the outermost grids.

\begin{tabular}{|c|c|c|c|}
\hline \multirow{2}{*}{ Designation } & \multicolumn{3}{|c|}{ Grid spacing (km) } \\
\cline { 2 - 4 } & Domain 1 & Domain 2 & Domain 3 \\
\hline 8-km & 24 & 8 & \\
6-km, 2-km & 18 & 6 & 2 \\
4-km & 36 & 12 & 4 \\
3-km, 1-km & 9 & 3 & 1 \\
\hline
\end{tabular}

Table 1. Designations used for different domain configurations. As the moving nest feature is used for these simulations, some grids are produced by the same model simulation (such as 6- and 2-km run).

The grid spacing and domain configuration chosen for each run are outlined in table 1 . In all simulations, 1-way nested domains are present, with the WRF vortex-tracking moving nest feature used on all inner nests. All grids are similar in their spatial extent, but different grid spacing is used, with the vertical grid spacing being held constant at 42 half- $\sigma$ levels.

In order to more clearly isolate differences between the simulations arising from resolution, a subset of model output variables is azimuthally- averaged. This is accomplished by averaging all points within $304 \mathrm{~km}$ of the storm center, which is defined as the local minimum of sea-level pressure, in concentric rings of $8-\mathrm{km}$ width. These averaged variables are then temporally averaged from forecast hours 60 to 72 , at a time when the simulated Ivan had reached full intensity in the model. This process also enables difference fields to be computed in a stormrelative sense for each simulation after averaging is performed.

\section{RESULTS}

The hurricane structure and intensity on the innermost domain exhibits substantial sensitivity to resolution. First, intensity differences will be discussed, followed by analysis of the symmetric, or system-averaged, fields.

\subsection{Intensity}

Figure 1 presents time series of minimum central pressure of Ivan, along with the National Hurricane Center (NHC) best track data. The 1$\mathrm{km}$ simulation exhibits the lowest minimum central pressure, $898 \mathrm{hPa}$, followed by the $2-\mathrm{km}$ run, which reaches $904 \mathrm{hPa}$. The $3-\mathrm{km}$ run, at $913 \mathrm{hPa}$, comes the closest to the best track data during Ivan's most intense phase, which was $\sim 910 \mathrm{hPa}$. The lowest central pressures for the 8, 6-, and 4-km simulations are all noticeably weaker, at 927, 923, and $926 \mathrm{hPa}$, respectively.

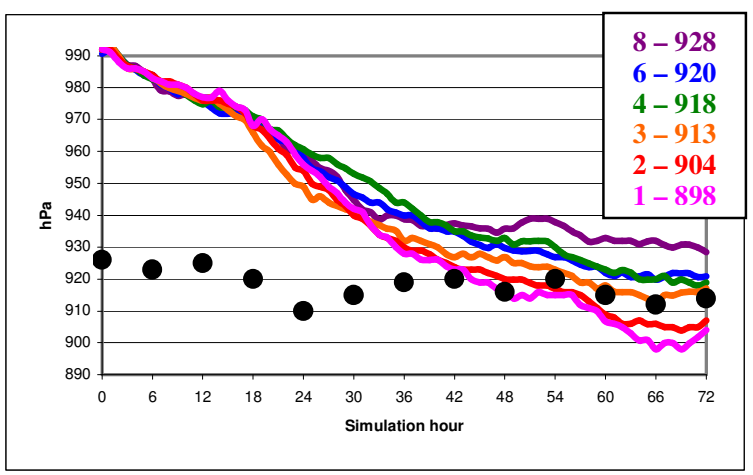

Fig. 1. Minimum central pressure as a function of time for the 8-km (purple), 6-km (blue), 4-km (green), 3-km (orange), 2-km (red), and 1-km (pink) runs, plotted with best track observations (black) from the NHC (Stewart 2006). Lowest central pressure for each run is shown in the inset box. 
Overall, a robust trend of increasing intensity with increasing resolution is found. However, the magnitude of the lowering of central pressure with decrease in grid spacing varies across the spectrum of simulations. The smallest increase in intensity per increase in resolution is seen as grid spacing drops from 6 to $4 \mathrm{~km}$, only resulting in a lowest central pressure drop of $2 \mathrm{hPa}$. But another decrease in grid spacing between the 4and $2-\mathrm{km}$ runs leads to a deepening of $18 \mathrm{hPa}$.

The system-averaged variables are next examined in order to see how the physical processes important to TC intensification change as the grid spacing decreases. .
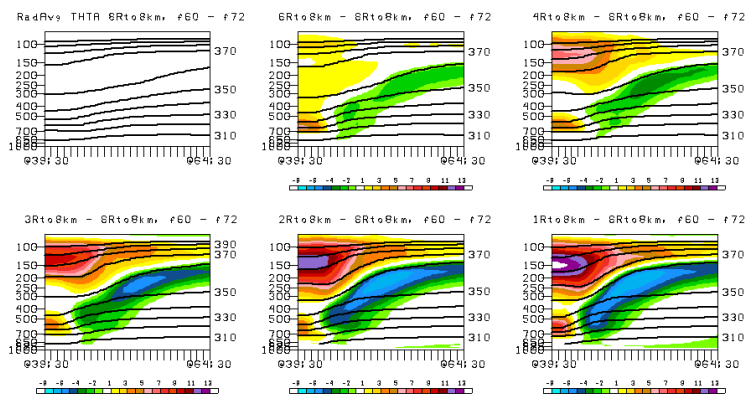

Fig. 2. Azimuthal and time averaged cross-sections of potential temperature $(\mathrm{K})$, all averaged to $8-\mathrm{km}$ grid spacing, shown from the center of the storm to $200 \mathrm{~km}$ away. Plotted every $10 \mathrm{~K}$ are the $8-\mathrm{km}$ simulation (top left), the $6-\mathrm{km}$ simulation (top center), 4-km simulation (top right), 3-km simulation (bottom left), 2-km simulation (bottom center), and 1-km simulation (bottom right). The difference plots between each simulation and the 8-km simulation are contoured and filled every $1 \mathrm{~K}$. Warmer (cooler) colors correspond to warmer (cooler) temperatures in the higher resolution runs, relative to the $8-\mathrm{km}$ run.

\subsection{Warm-core}

In the warm-core TC vortex, air ascends in the eyewall, releasing latent heat as the vapor within rising air parcels condenses. This heat is ventilated in the upper troposphere, with outflow and divergence in the upper troposphere as well as subsidence in the eye of the storm. This subsidence causes further warming in the eye through compressional heating, hydrostatically lowering the surface pressure. Here, the $\theta$ fields are examined to see if the more intense storms in the higher resolution simulations do have more a warmer warm-core (Fig. 2). It is apparent from the difference fields that higher resolution simulations do have warmer cores; however, a significant amount of warming is found above
$250 \mathrm{hPa}$, with a slight warming signature centered at $600 \mathrm{hPa}$. Overall, there is a noticeable lack of warming, and a slight signature of cooling, in the mid-levels.

\subsection{Magnitude of the Horizontal Wind}

Another implication of the TC being a warm-core vortex is related to the slope of the eyewall. The gradient thermal wind relation dictates that, in a warm-core system, cyclonic flow decreases with height. As the inward-directed pressure gradient force weakens, the horizontal wind gradient lessens and the eyewall leans out further with height. However, in both sets of simulations, as the resolution increases, there is a significant increase in the vertical extent of the primary circulation. In the 1- and 2-km simulations, 60 $\mathrm{ms}^{-1}$ winds extend up to $300 \mathrm{hPa}$ (Fig. 3). In the $3-\mathrm{km}$ run, this contour extends up to only 500 $\mathrm{hPa}$, and the coarser resolution simulations $(8,6$, and 4-km) all place this contour around $700 \mathrm{hPa}$ and below.

The lack of significant warming below $300 \mathrm{hPa}$ in the higher resolution simulations is consistent with these runs having TC eyewalls with less weakening of the cyclonic winds with height. Most of the warming in the higher resolution simulations occurs above $300 \mathrm{hPa}$ and this level is also where the cyclonic winds begin to lessen significantly with height for grid spacing of $2-\mathrm{km}$ and below. This horizontal wind structure would also imply that, as resolution increases, the eyewall of TC should lean out less with height.
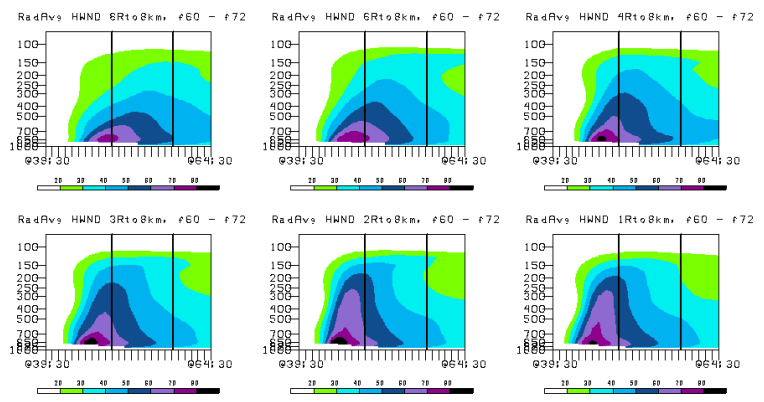

Fig. 3. As before, but with the magnitude of the horizontal wind (every $10 \mathrm{~ms}^{-1}$ from 20 to $80 \mathrm{~ms}^{-1}$ ). Winds $60 \mathrm{~ms}^{-1}$ and above are denoted by the light purple color. Vertical lines are shown every $75 \mathrm{~km}$ from the center. 


\subsection{Vertical Motions}

Here, the vertical wind is divided and averaged into ascent and descent, in order to examine the structure of the main eyewall updraft and of the subsidence in the TC core. The system-averaged ascent indicates significant differences in eyewall updraft speed and structure as resolution increases (Fig. 4). The 2-km simulation has the strongest eyewall updraft, with the average ascent throughout the depth of eyewall updraft being greater than $2 \mathrm{~ms}^{-1}$ in all simulations with grid spacing less than 4-km, except the 1-km run. Also, the shape of the eyewall shifts as resolution increases. All simulations below 4-km have a distinctively more upright eyewall in the midlevels, with some sloping of the eyewall outward below $700 \mathrm{hPa}$ and above $200 \mathrm{hPa}$.
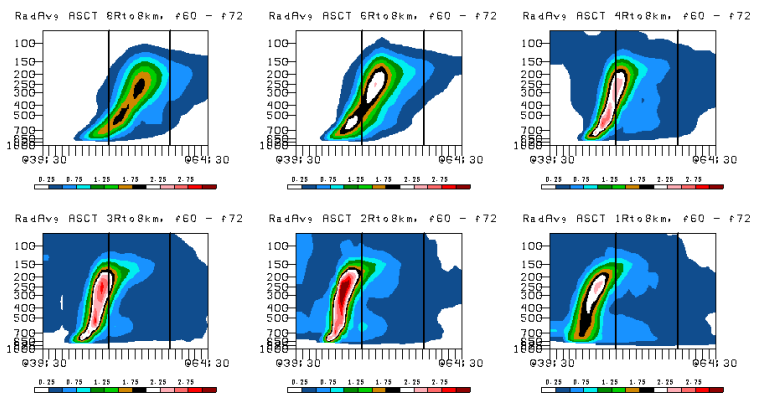

Fig. 4. As before, but with positive vertical motion (every $0.25 \mathrm{~ms}^{-1}$ ) for the 8- (top left), 6- (top center), 4- (top right), 3- (bottom left), 2- (bottom center), and 1-km (bottom right) simulations. The green contour denotes ascent greater than 1 $\mathrm{ms}^{-1}$. The white contour denotes ascent greater than $2 \mathrm{~ms}^{-1}$.

One outlier in this trend of increasing updraft speed with increasing resolution is the $1-\mathrm{km}$ simulation. There is a significant reduction of the averaged updraft speed as grid spacing is reduced from $2 \mathrm{~km}$ to 1 . As previously discussed, the finding by Bryan that finer resolution simulations have the ability to become more turbulent implies that sufficiently fine resolution could lower updraft speeds, because intra-eyewall processes detrimental to updraft speed (such as entrainment) could be partially-resolved. This hypothesis is further implied by the full vertical motion field at $850 \mathrm{hPa}$ (Fig. 5). While the 3and $2-\mathrm{km}$ runs show relatively smooth ascent surrounding the eye, in the $1-\mathrm{km}$ simulation the eyewall contains numerous up and downdrafts that are smaller in spatial scale and much larger in magnitude. Overall, there is a remarkable increase in the number of both up and downdraft cores in the $1-\mathrm{km}$ run.

Figure 6 shows averaged downward vertical motions. Overall, as grid spacing decreases, both the areas of subsidence within the TC eye and spreading out from the lower eyewall are stronger, indicating that downdrafts in the eyewall, as well as subsidence within the eye, are better resolved. However, the area of the strongest descent does shift as resolution increases. The 6-km run shows equally strong values of descending vertical motion in the upper levels of the eye and outside the eye, beneath the eyewall. In contrast, the 1-km simulation clearly has the strongest downdrafts placed around 700 $\mathrm{hPa}$, just inside the main eyewall updraft. Overall, there is a robust trend of strong subsidence at the base of the TC, both underneath the eyewall and within the eye.

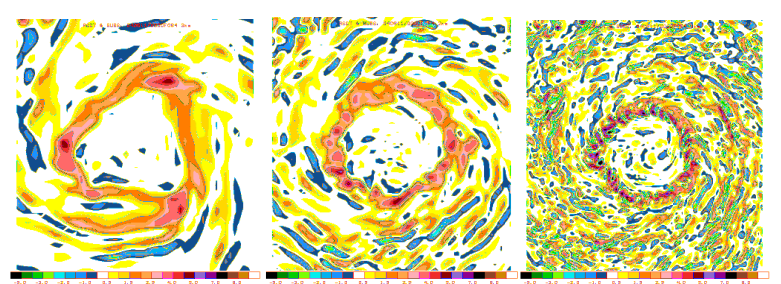

Figure 5. $850-\mathrm{hPa}$ vertical wind component at simulation time 6430 contoured $0.5 \mathrm{~ms}^{-1}$ up to $5 \mathrm{~ms}^{-1}$ and every $1 \mathrm{~ms}^{-1}$ thereafter for the 3- (left), 2- (center), and 1-km runs (right), with positive vertical motion in warmer colors and negative vertical motion in cooler colors.
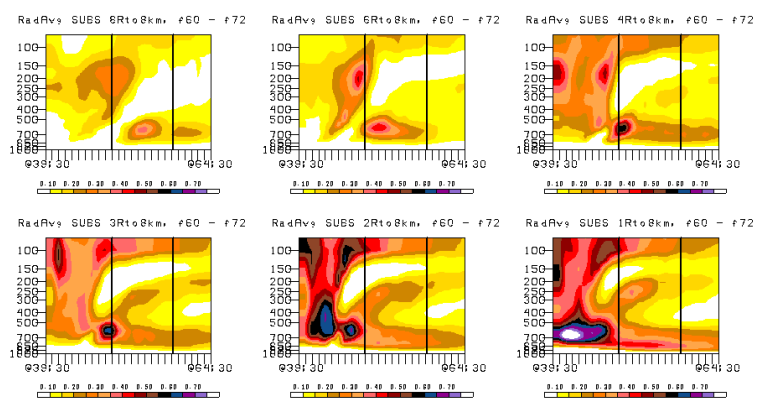

Fig. 6. As in Fig. 4, but with downward vertical motion (every $0.05 \mathrm{~ms}^{-1}$. The black contour denotes subsidence greater than $0.55 \mathrm{~ms}^{-1}$. The purple contour denotes subsidence greater than $0.70 \mathrm{~ms}^{-1}$.

\subsection{Asymmetric features}

Examination of high temporal resolution model output reveals the characteristics of the potential vorticity (PV) features changes dramatically as resolution increases (Fig. 7). While the 8- and 6$\mathrm{km}$ simulations show a quiescent PV ring, the 
higher resolution simulations have PV maxima that are smaller in size and larger in magnitude as grid spacing decreases. Also, in the 2- and 1-km runs, some PV anomalies are shown breaking off from the high-PV eyewall region and entering the low-PV environment of the eye. These events also occur in the 3-and 4- $\mathrm{km}$ simulations, but are not shown. An important asymmetric feature, VRWs propagate along PV gradients. When counter-propagating VRWs on the inside and outside edge of the eyewall phase-lock and break, mixing PV from the eyewall into the eye. Additionally, there are polygonal eyewall segments in 4-km and high resolution simulations, which is also indicative of breaking VRWs (Schubert et al. 1999).

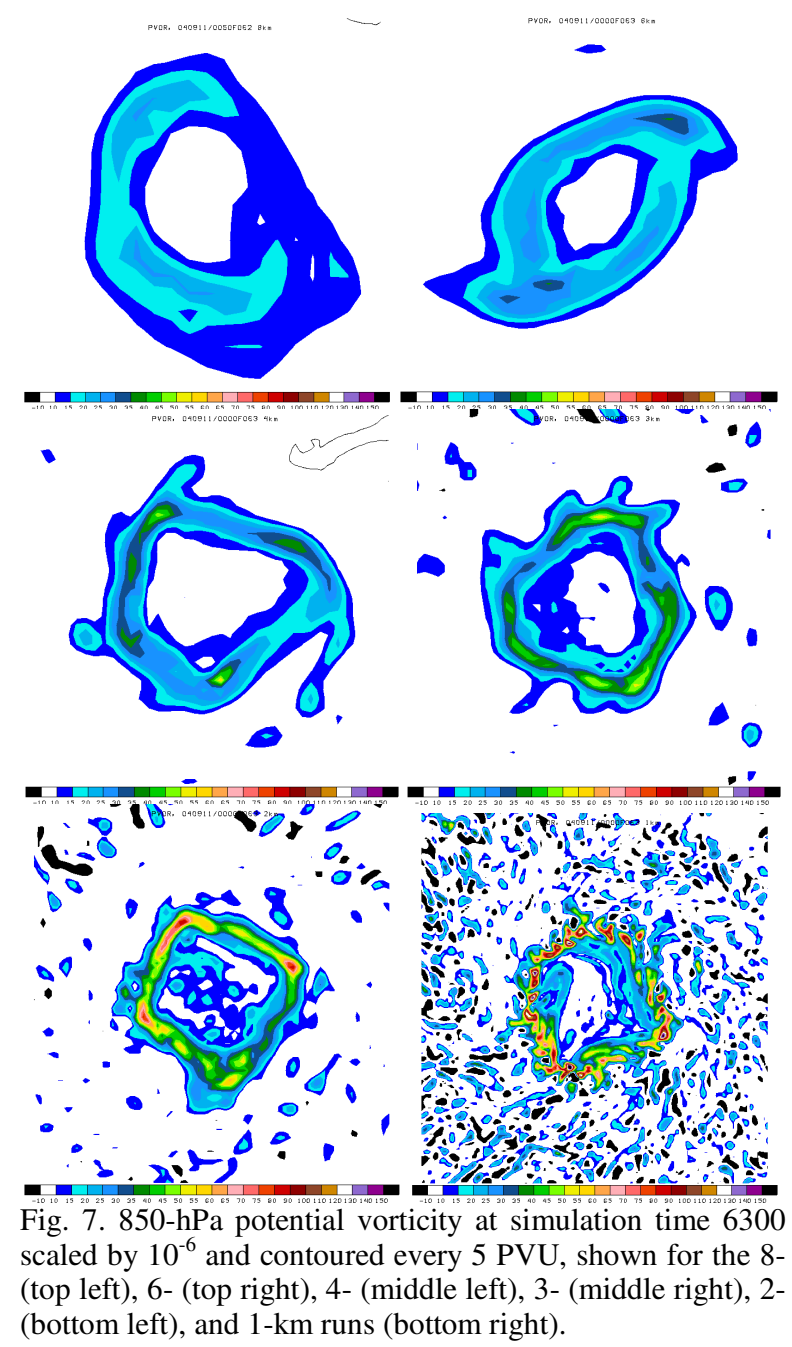

\section{DISCUSSION}

As discussed previously, there is little difference in minimum central pressure between grid spacing between 8 and 4-km. However, significant deepening occurs as grid spacing below $4 \mathrm{~km}$ is used. Also, these model runs show a shift in the structure of the eyewall. The reduced slope of the eyewall with height, as well as the deep layer of strong horizontal wind speed all suggest a lack of warming in the mid-levels in the finer resolution simulations. This would be consistent with a reduction in the anticyclonically-directed thermal wind, which would result in less anticyclonic shear in the midlevels of the TC (Fig. 3). Indeed, plots of systemaveraged $\theta$ show a reduction in the warming of the finer simulations in the middle levels of the eye (Fig. 2). This suggests some sort of ventilation mechanism acting to remove high- $\theta$ air from the eye in the runs with smaller grid spacing.

In accordance with hydrostatic balance, it is expected that a TC of a lower minimum central pressure should also have a warmer eye. As air descends within the eye, it warms through adiabatic compression. When this warm dry air reaches the bottom of the eyewall, it is moistened by fluxes from the ocean's surface, making the bottom of the eyewall a reservoir of high $\theta_{\mathrm{e}}$ air. A simulation with sufficient resolution could somewhat resolve the phase-locking and breaking of VRWs. This ventillation mechanism could mix high $\theta$ air into the eyewall, where it could be used to fuel buoyant eyewall updrafts (Schubert et al. 1999, Persing and Montgomery 2003).

As resolution increases, subsidence within the eye also increases, with maxima at the $700 \mathrm{hPa}$ level, both beneath the eyewall updraft and within the eye. Such pronounced subsidence at the bottom of the eye could be induced by the divergence of air at the bottom of the eyewall, as VRWs act as to ventilate the bottom of the eye. Converging air from above would be further adiabatically-compressed and warmed (Willoughby 1998). Also, the divergence associated with the ventillation of the eye would aid intensification by further dropping the central pressure of the TC. Such an additional source of intensification could explain why the $1-\mathrm{km}$ 
simulation has such a weak eyewall updraft but remains the most intense simulation.

Mixing between the eye and eyewall has been discussed as an important process in TC intensification. However, the mixing of lowmomentum eye air into the eyewall would act to slow winds within the eyewall, and could be a physical process that would spin down the vortex. Therefore, a simulation in which VRWs are somewhat resolved and individual convective updrafts are poorly resolved might experience more of the spin down effect, as low-momentum, high $\theta$ air from the eye would act to slow the primary circulation, but would be under-utilized by the poorly resolved convective updrafts. Such a condition could explain why the 4-km simulation did not experience the rapid deepening of the 2-km simulation, but did exhibit evidence of breaking VRWs mixing PV between the eye and eyewall (Fig. 7).

\section{CONCLUSIONS}

In this study, the sensitivity of physical processes to grid spacing has been discussed, with the goal of suggesting the appropriate grid spacing to be used for other studies. It is found that little intensity change occurs at as resolution is increased from 8 to $6 \mathrm{~km}$. Also, the TC structure changes little at grid spacings of 8 and 6 . Therefore, changes in resolution at these grid spacings do not affect the model's solution either in TC intensity or structure. As the grid spacing drops to $4 \mathrm{~km}$, while there is little increase in TC intensity, the physical processes simulated do appear to change, with polygonal eyewall segments appearing as possible evidence of VRWs. At 2-km, there is a significant drop in central pressure. Also, polygonal eyewall structures and localized, intense updraft cores appear more frequently. At 1-km grid spacing, not only do an ensemble of eyewall updraft cores appear, but also downdraft cores appear within the eyewall.

If the results of this case can be generalized, it is suggested that for simulations where it is only necessary to realistically simulate the TC vortex, $8-\mathrm{km}$ is sufficient for this task. However, if features on the scale of the eyewall itself are to be studied, 4-km is the coarsest resolution that should be used. At a grid spacing of $2 \mathrm{~km}$, features within the eyewall, i.e. an ensemble of updraft cores within the eyewall, appear to be somewhat resolved. However, only at $1-\mathrm{km}$ grid spacing, are both updraft and downdraft cores within the eyewall partially resolved.

\section{FUTURE WORK}

Future work could include additional cases of real hurricanes and possibly an idealized case in order to isolate any case-dependent conclusions from this study and increase the robustness of results. However, the most valuable additional work would involve extending these results over a greater number of possible grid spacings.

\section{ACKNOWLEDGEMENTS}

This research was supported by NSF grants ATM-0334427 and ATM-0603760, and DOE grant ER64448, all awarded to North Carolina State University. The authors would also like to thank RENCI for making available their computing resources and technical support.

\section{REFERENCES}

Bryan, G. H., J. C. Wyngaard, and J. M. Fritsch, 2003: Resolution requirements for the simulation of deep moist convection. Mon. Wea. Rev., 131, 23942416.

Cram, T. A., J. Persing, M. T. Montgomery, S. A. Braun, 2007: A Lagrangian trajectory view on transport and mixing processes between the eye, eyewall, and environmental using a high-resolution simulation of Hurricane Bonnie (1998). J. Atmos. Sci., 64, 1835-1856.

Emanuel, K. A., 1986: An air-sea interaction theory for tropical cyclones. Part I: Steady state maintenance. J. Atmos. Sci., 43, 585-604.

Janjić, Z. I., 1994: The step-mountain Eta coordinate model: Further development of the convection, viscous sublayer and turbulent closure schemes. Mon. Wea. Rev., 122, 927-945.

Janjić, Z. I., 2002: Nonsingular implementation of the Mellor-Yamada Level 2.5 Scheme in the NCEP Meso model. NCEP Office Note, No. 437, 61 pp

Kain, J. S., and J. M. Fritsch, 1993: Convective parameterization for mesoscale models: The KainFritsch scheme. Cumulus Parameterization, 
Meteor. Monogr., No. 46, Amer. Meteor. Soc., 165-170.

Ooyama, , K. V., 1982: Conceptual evolution of the theory and modeling of the tropical cyclone. $J$. Roy. Meteor. Soc. Japan, 60, 369-379.

Persing, J. and M. T. Montgomery, 2003: Hurricane superintensity. J. Atmos. Sci., 60, 2349-2371.

Rogers, R., S. Aberson, M. Black, P. Black, J. Cione, P. Dodge, J. Dunion, J. Gamache, J. Kaplan, M. Powell, N. Shay, N. Surgi, and E. Uhlhorn, 2006: The Intensity Forecasting Experiment: A NOAA Multiyear Field Program for Improving Tropical Cyclone Intensity Forecasts. Bull. Amer. Meteor. Soc., 87, 1523-1537.

Rotunno, R. and K. A. Emanuel, 1987: An air-sea interaction theory for tropical cyclones. Part II: Evolutionary study using a nonhydrostatic axisymmetric numerical model. J. Atmos. Sci., 44, 542-561.

Schubert, W. H., M. T. Montgomery, R. K. Taft, T. A. Guinn, S. R. Fulton, J. P. Kossin, and J. P. Edwards, 1999: Polygonal eyewalls, asymmetric eye contraction, and potential vorticity mixing in hurricanes. J. Atmos. Sci., 56, 1197-1223.

Stewart, S. R., cited 2006: National Hurricane Center Tropical Cyclone Report: Hurricane Ivan. [Available from http://www.nhc.noaa.gov/2004ivan.shtml]

Thiébaux, J, E Rogers, W Wang, and B Katz, 2003: A new high-resolution blended real-time global sea surface temperature analysis. Bull. Amer. Meteor. Soc., 84, 645-656

Weisman, M. L., W. C. Skamarock, and J. B. Klemp, 1997: The resolution dependence of explicitly modeled convective systems. Mon. Wea. Rev., 125, 527-548.

Willoughby, H. E., 1998: Tropical cyclone eye thermodynamics. Mon. Wea. Rev., 126, 30533067.

Yang, B., Y. Wang, and B. Wang, 2007: The effect of internally generated inner-core asymmetries on tropical cyclone potential intensity. J. Atmos. Sci., 64, 1165-1188. 